\title{
Análisis del estrés y las exigencias cinemáticas y fisiológicas de los bomberos durante la jornada laboral
}

\section{Analysis of stress and kinematical and physiological demands during firefighters workday}

\author{
J. Romero-Sánchez ${ }^{1}$, C. Gómez-Carmona², A. Bastida-Castillo ${ }^{3}$ J. Pino-Ortega ${ }^{4}$ \\ 1 Graduado en Ciencias de la Actividad Física y el Deporte. Facultad de Ciencias de la Actividad Física y el Deporte. \\ Universidad de Murcia, San Javier, Murcia (España). \\ 2 Doctorando en Ciencias del Deporte. Departamento de Actividad Física y Deporte. Facultad de Ciencias de la Actividad Física y el Deporte. \\ Universidad de Extremadura, Cáceres (España). \\ 3 Doctorando en Ciencias del Deporte. Departamento de Actividad Física y Deporte. Facultad de Ciencias de la Actividad Física y el Deporte. \\ Universidad de Murcia, San Javier, Murcia (España). \\ 4 Profesor y Doctor en Ciencias del Deporte. Departamento de Actividad Física y Deporte. Facultad de Ciencias de la Actividad Física y el Deporte. \\ Universidad de Murcia, San Javier, Murcia (España).
}

\begin{abstract}
Resumen: El trabajo de los bomberos es considerado como una de las profesiones con mayor exigencia física y predisposición a padecer estrés, debido a las exigentes condiciones de trabajo y la responsabilidad sobre vidas humanas. Los objetivos de la presente investigación fueron: (i) describir las exigencias cinemáticas, fisiológicas y de estrés en bomberos, y (ii) comparar estas exigencias en función de los años de experiencia. Ocho bomberos del parque del Ayuntamiento de Cartagena participaron voluntariamente, siendo divididos en dos grupos en función de la experiencia: (1) experimentados: $>12$ años $(n=4)$; (2) noveles: $<7$ años $(n=4)$. Se registraron las variables aceleración total (AcelT), frecuencia cardíaca media (FCmedia) y ratio simpático-parasimpático (R-S|Ps). Los resultados mostraron diferencias entre actividades, encontrando los mayores valores en deporte y salidas. En el análisis inter-grupos, se encontraron diferencias en las variables AcelT y FCmedia en deporte, siendo mayor en los bomberos experimentados $(p<.05) ; y$ en la variable R-S|Ps en Práctica y Salida con urgencia $(p<.05)$, siendo mayor en los noveles. En conclusión, los bomberos con menos experiencia sufren un mayor estrés durante la jornada laboral. Por tanto, es necesario la incorporación de personal cualificado para diseñar una planificación específica del entrenamiento y de las estrategias de afrontamiento ante la resolución de conflictos adaptadas a la situación real.
\end{abstract}

Palabras claves: bomberos, estrés laboral, variabilidad de la frecuencia cardiaca. Abstract: The work of firefighters is considered one of the professions with greater physical demands and predisposition to suffer stress, due to the hazardous working conditions and the responsibility with human lives. The aims of the present study were to: (i) describe the kinematical, physiological and stress demands of firefighters, and (ii) compare these demands in relation to the experience years. Eight firefighters from the fire department of Cartagena participated voluntarily, dividing them into two groups: (1) experienced: $>12$ years $(n=4)$; (2) novels: $<7$ years $(n=4)$. The registered variables were total acceleration (AcelT), heart rate average (HRavg) and sympathetic-parasympathetic ratio (R-S|Ps). The results shown differences between activities, finding the highest values in sport and exits. In the between-groups comparison, differences in AcelT and HRavg in sport $(p<.05)$, being higher in experienced group; and differences in R-S|Ps in practice and exits with urgency $(p<.05)$, being higher in novels group. In conclusion, the firefighters with less experience suffer greater stress during workday. Therefore, it is necessary the incorporation of qualified staff to design specific training and coping strategies before the resolution of conflicts adapted to the real situation.

Keywords: firefighters, work stress, heart rate variability.

\section{Introducción}

Los servicios de seguridad y emergencia tienen como función hacer frente a situaciones excepcionales que suponen grandes riesgos. Por lo tanto, las personas que se ocupan de tales servicios deben afrontar hechos y entornos de trabajo que sobrepasan las experiencias laborales de la mayoría de los seres humanos. Dentro de estos servicios, se distinguen 4 grupos siguiendo la clasificación realizada por Guidotti (2001): servicio de policía y fuerzas armadas, trabajadores que utilizan

Dirección para correspondencia [Correspondence address]: Carlos David Gómez Carmona. Universidad de Extremadura, Facultad de Ciencias del Deporte. Avenida de la Universidad s/n. Cáceres (Espańa) Email: cdgomezcarmona@gmail.com sustancias y preparados peligrosos, personal sanitario y paramédicos y, finalmente, servicio de lucha contra incendios. Entre ellos se puede diferenciar entre acciones preventivas, llevadas a cabo con el objeto de prevenir una situación de peligro o riesgo, y acciones reactivas, las cuales tienen la finalidad de actuar sobre el peligro lo antes posible para minimizar los daños. Los distintos servicios de seguridad y emergencia ya citados no se clasifican en preventivos o reactivos, sino que cada uno de ellos puede realizar las dos acciones indistintamente, aunque por norma general realice con más frecuencia una de ellas (Guidotti, 2001). De esta forma, y en referencia al subgrupo de servicio de lucha contra incendios, según la Real Academia Española (2014), el cuerpo de bomberos está 
formado por operarios que realizan funciones de extinción de incendios. Sin embargo, De Vicente (2005) señala otro tipo de intervenciones: salvamento, rescates, emergencias, evacuación de personas, actividades de prevención, asistencia técnica y acciones divulgativas, entre otras actividades.

En Espańa el servicio de bomberos del Estado puede depender de la Comunidad Autónoma, del Ayuntamiento o de la Diputación pertinente (De Vicente, 2005). El parque de bomberos de Cartagena está regulado por el Ayuntamiento de esta ciudad, contando con 107 bomberos en su plantilla, entre los que se podrían distinguir diferentes rangos: sargentos, cabos, bomberos conductores o bomberos control. Todos ellos son funcionarios del Ayuntamiento de Cartagena y forman el conjunto de trabajadores del parque de bomberos junto con el personal de la administración, jefatura y el personal del servicio de taller mecánico. Cada uno de estos puestos de trabajo posee una regulación propia debido a sus diferencias legislativas. Esto implica que las características de cada organización son variables y, por lo tanto, hace muy difícil la comparación entre los cuerpos de bomberos existentes en España. Sin embargo, se puede decir que las condiciones físicas y psicológicas que requiere un bombero son muy similares en cada una de estas clasificaciones, pues todos se exponen a grandes riesgos en sus puestos de trabajo.

Las condiciones en dicho trabajo tienen repercusiones sobre su salud (Laca, Mejía, y Condra, 2014; Topa, Fernández, y Lisbona, 2005). Según Salvador y González-Bono (1995) una de las fuentes principales de estrés en la sociedad occidental es el ámbito laboral. La labor de los bomberos somete a estos a una extenuante y constante exigencia de sus capacidades físicas (Avellaneda-Pinzón y Urbina, 2015; Lara, García, Torres-Luque, y Zagalaz, 2013; López et al., 2006; Prieto et al., 2010; Sabido, Gómez, Barbado, y Gómez-Valadés, 2013), como también de las psicológicas (Curilem, Almagià, Yuing, y Rodríguez, 2014; Moreno, Morett, Rodríguez, y Morante, 2006; Peñacoba, Díaz, Goiri, y Vega, 2000). Según un estudio realizado por Moya-Albiol, Serrano, González-Bono, Rodríguez-Alarcón, y Salvador (2005), durante el tiempo de trabajo se puede observar una mayor percepción de estrés, un estado de ánimo más negativo y mayor frecuencia cardiaca que cuando no se trabaja. Otro estudio realizado por Gómez-Alcaina, Montero-Marín, Demarzo, Pereira, y García-Campayo (2013) nos indica que podemos encontrar biomarcadores relacionados con el estrés y el burnout a nivel cardiovascular como: tensión arterial, frecuencia cardiaca y variabilidad de la frecuencia cardiaca.

Cualquier trabajador puede padecer estrés en algún momento de su vida laboral, no obstante, existen ciertas profesiones con mayor predisposición a padecerlo: médicos, enfermeros, policías, bomberos, controladores aéreos, mineros, artificieros, etc. (Cayo y Chamorro, 2015). Aunque parece más común encontrar estudios relacionado con el estrés en otras profesiones (García, Fantin, y Pardo, 2016; Paris, 2016) sigue llamando la atención la escasez de trabajos dedicados a los bomberos. Rotger (1997) identifica varias fuentes de estrés en el trabajo del bombero, entre ellas destaca: las condiciones de trabajo arriesgadas, las presiones para que se realice con rapidez, la necesidad de tomar muchas decisiones, la responsabilidad sobre vidas humanas y la fatiga resultante de un esfuerzo físico importante, entre otras. A partir del conocimiento de las exigencias de los deportistas, programas específicos de entrenamiento (Mateo-March et al., 2013) y diferentes estrategias de afrontamiento (Molinero, Salguero, y Márquez, 2012) han conseguido reducir el estrés en deportistas. Por lo tanto, el objetivo de este estudio es identificar el nivel de estrés y exigencias físicas durante la jornada laboral típica de un cuerpo de bomberos y comprobar si existen diferencias con la experiencia y las diferentes fases de la jornada sobre estos niveles.

\section{Método}

\section{Participantes}

8 sujetos varones pertenecientes al cuerpo de bomberos del Ayuntamiento de Cartagena participaron voluntariamente en este estudio, los cuales presentaban las siguientes características antropométricas $(\mathrm{Edad}=45,5 \pm 7,8$ años; Peso $=77,5$ \pm 4,72 Kg; Altura $=176 \pm 4,87 \mathrm{~cm}$; Experiencia $=9,5 \pm 9,61$ ańos). Todos los participantes firmaron un consentimiento informado previamente al inicio de la investigación. La investigación fue realizada siguiendo los principios de ética de investigación de la declaración de Helsinki (2013), siendo aprobado por el comité de bioética de la Universidad de Murcia. Además, se obtuvo una autorización del jefe del cuerpo de bomberos y del Ayuntamiento de Cartagena.

\section{Procedimiento}

Se analizó un día completo de servicio ( 24 horas) de 8 bomberos pertenecientes al Parque de Bomberos de Cartagena el día 5 de mayo de 2017. En función de la experiencia de los trabajadores, estos se dividieron en dos grupos de 4 integrantes: (1) experimentados (experiencia superior a 12 ańos) y (2) noveles (experiencia inferior a 7 ańos). Durante la duración, se clasificaron las distintas actividades en cinco grupos: (a) Dormir (entendido como el periodo en el cual los bomberos se encontraban en un estado de suspensión de los sentidos y todo movimiento voluntario); (b) Reposo (referido a periodos de poca actividad como pueden ser comer, ver la televisión, estudiar, etc.); (c) Prácticas, alusivo al tiempo donde los bomberos realizan el periodo de prácticas o simulacros sobre protocolos de actuación; (d) Deporte (referido al periodo en el que el sujeto realiza cualquier práctica deportiva o entrena- 
miento); (e) Salida 1 (entendiendo esta como una salida sin extrema urgencia, como podría ser la limpieza de manchas de aceite en la carretera o retirada de panales de abejas de la vía pública) y, por último, (f) Salida 2 (relacionado con las salidas de urgencia, como incendios o accidentes de tráfico).

\section{Material}

Características antropométricas

La altura de los sujetos fue medida con un tallímetro de pared (SECA, Hamburgo, Alemania). El peso corporal de los sujetos se obtuvo mediante un monitor de composición corporal modelo BC-601 (TANITA, Tokio, Japón).

\section{Carga externa y carga interna}

Se utilizaron 16 dispositivos inerciales (Wireless Inercial Movement Unit) denominado WIMU PRO ${ }^{\mathrm{TM}}$ (RealTrack Systems, Almería, España) el cual integra diferentes sensores (acelerómetros, giróscopos, un magnetómetro, GPS, UWB, entre otros), el cual ha mostrado una alta fiabilidad y validez para el posicionamiento tanto en condiciones de exterior (Bastida-Castillo et al., 2018a) e interior (Bastida-Castillo et al., 2018b). Este dispositivo tiene una frecuencia de muestreo de $100 \mathrm{~Hz}$. Los dispositivos tienen una autonomía de 4 horas aproximadamente que no es suficiente para registrar un día completo. Por lo tanto, se utilizaron dos dispositivos para cada bombero los cuales se intercambiaban cada dos horas y media de servicio, manteniendo constante la monitorización de los participantes. Para la realización de este estudio, los datos fueron grabados en la memoria interna del dispositivo. Para anexionar el dispositivo a los bomberos, este se introducía en un arnés diseñado específicamente para incorporarlo y posteriormente se colocaba a cada bombero (figura 1).
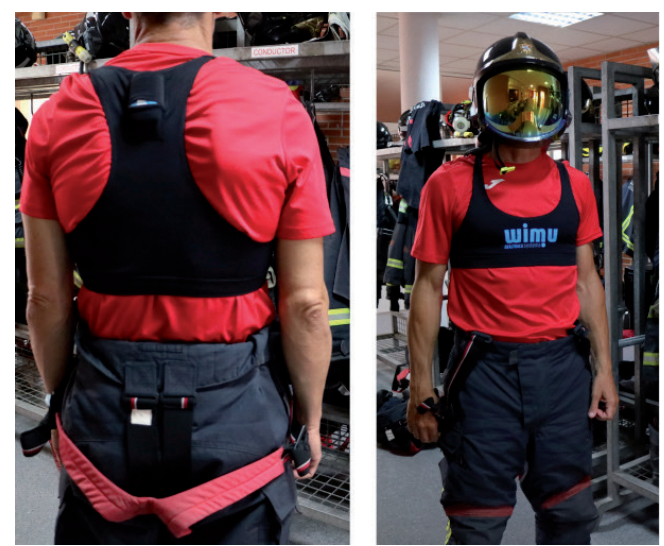

Figura 1. Adhesión del dispositivo WIMU en el arnés específico y colocación del mismo en uno de los sujetos participantes en la investigación.
Para monitorizar la frecuencia cardiaca de los bomberos se empleó una banda GARMIN ${ }^{\mathrm{TM}}$ (Garmin Ltd., Olathe, Kansas, Estados Unidos) la cual enviaba los datos al sistema WIMU PRO ${ }^{\mathrm{TM}}$ a través de tecnología inalámbrica Ant+ con una frecuencia de muestreo de $4 \mathrm{~Hz}$ (Molina-Carmona, Gómez-Carmona, Bastida Castillo, y Pino-Ortega, 2018). Para conocer el momento exacto en el que se realizaban las distintas actividades, el encargado de la grabación portaba un pulsador. $\mathrm{Al}$ accionar dicho pulsador este envía una señal inalámbrica mediante tecnología Ant+ al dispositivo WIMU $\mathrm{PRO}^{\mathrm{TM}}$ y esta señal queda registrada en la línea temporal de la grabación del aparato. Además, se realizaba una anotación en el cuaderno de registro de la hora, la actividad y el sujeto que la realizaba. Para extraer la información de la memoria interna de los dispositivos inerciales y analizar las diferentes variables se utilizó el software SPRO ${ }^{\mathrm{TM}}$ (RealTrack Systems, Almería, España). Los datos originales de la monitorización fueron distribuidos en intervalos de $10 \mathrm{~min}$.

Variables

Aceleración total (AcelT): La aceleración total registrada. Ésta es la suma vectorial de los ejes del acelerómetro (Kunze, Bahle, Lukowicz, y Partridge, 2010; O’Donovan, Kamnik, O’Keeffe, y Lyons, 2007; Gómez-Carmona, Bastida-Castillo, García-Rubio, Ibáńez, y Pino-Ortega, 2018), donde (z) aceleración antero-posterior, (x) aceleración medio-lateral e (y) aceleración vertical.

$$
\text { AcelT }=\sqrt{x^{2}+y^{2}+z^{2}}
$$

Frecuencia cardiaca (FC): Representa el número de contracciones del corazón por unidad de tiempo. Se mide en pulsaciones por minuto y es una clara respuesta fisiológica al aumento de la actividad (Bouzas, Ottoline, y Delgado, 2010).

Ratio simpático - parasimpático $\left(R-S \mid P_{S}\right)$ : Aporta información del equilibrio entre ambos sistemas. Un equilibrio ideal dará lugar a valores bajos, lo que denotará en una alta actividad parasimpática y bajos valores de actividad simpática, es decir, el organismo estará trabajando poco y a la vez su estado de estrés será bajo. Por el contrario, si se obtienen valores altos, esto reflejará que el organismo se encuentra en una situación de estrés (Rosales-Soto, Corsini-Pino, MonsálvesÁlvarez, y Yáńez-Sepúlveda, 2016).

Análisis estadístico

Para determinar la distribución de los datos se utilizó la prueba Shapiro-Wilk (Field, 2013). El análisis mostró una distribución no normal, por lo que se hicieron pruebas no paramétricas. Se realizó un primer análisis descriptivo, mostrando 
los resultados mediante los estadísticos media y desviación estándar. Para la comparativa entre los grupos analizados, diferenciado por actividades, en las variables AcelT, FC, y R-S|Ps se realizó la prueba estadística U de Mann-Whitney. Además, se calculó el tamaño del efecto de las diferencias usando el estadístico d de Cohen utilizando la plantilla facilitada por Hopkins (2010) (www.sportsci.com). Las pruebas estadísticas fueron realizadas mediante el software SPSS 24.0 (SPSS Inc. Chicago IL, EEUU). La significación estadística se estableció con el valor $\mathrm{p}<.05$.

\section{Resultados}

Los resultados reportados mostraron una media total de AcelT: $1.59 \pm 1.73$; FC: $75.39 \pm 12.19$; y R-S|Ps: $0.93 \pm 1.29$ durante una jornada completa. Siendo una media de AcelT: $1.87 \pm 1.86$; FC: $79.76 \pm 12.47$; . R-S|Ps: $1.01 \pm 1.32$ durante las fases que requieren movimiento de los participantes (prácticas, reposo, deporte, salida 1 y salida 2).

Salida 1 y salida 2 reportaron las mayores diferencias $(p<.05$ y $p<.001$ respectivamente) entre los grupos 1 y 2 (S1: $\mathrm{TE}=0.49 ; \mathrm{S} 2$ : $\mathrm{TE}=0.44)$. No se reportaron diferencias significativas en AcelT y sólo en salida 2 se reportaron diferencias significativas en R-S|Ps ( $p<.01)$ entre grupos (Tabla 1$)$.

Tabla 1. U de Mann-Whitney para la comparación de las variables en las distintas actividades.

\begin{tabular}{|c|c|c|c|c|c|c|c|c|c|}
\hline \multirow[b]{3}{*}{ Actividad } & \multirow[b]{3}{*}{ Dormir } & \multirow[b]{3}{*}{ Grupos } & \multirow[b]{3}{*}{1} & \multicolumn{2}{|c|}{ AcelT } & \multicolumn{2}{|c|}{$\mathrm{FC}$} & \multicolumn{2}{|c|}{$\mathrm{R}-\mathrm{S} \mid \mathrm{Ps}$} \\
\hline & & & & \multicolumn{2}{|c|}{$\mathrm{M} \pm \mathrm{DE}$} & \multicolumn{2}{|c|}{$\mathrm{M} \pm \mathrm{DE}$} & \multicolumn{2}{|c|}{$\mathrm{M} \pm \mathrm{DE}$} \\
\hline & & & & 0.58 & \pm 0.80 & 57.49 & \pm 10.81 & 0.66 & \pm 1.13 \\
\hline & & & 2 & 0.63 & \pm 1.00 & 65.75 & \pm 9.52 & 0.63 & \pm 1.16 \\
\hline & & \multicolumn{2}{|l|}{ Valor $\mathrm{p}$} & \multicolumn{2}{|c|}{ NS } & \multicolumn{2}{|c|}{$<0.001$} & \multicolumn{2}{|c|}{ NS } \\
\hline & & \multicolumn{2}{|l|}{ TE } & \multicolumn{2}{|c|}{-0.03} & \multicolumn{2}{|c|}{-0.38} & \multicolumn{2}{|c|}{0.01} \\
\hline & Reposo & Grupos & 1 & 1.34 & \pm 1.28 & 68.73 & \pm 10.71 & 0.95 & \pm 1.12 \\
\hline & & & 2 & 1.64 & \pm 2.57 & 75.28 & \pm 12.86 & 0.94 & \pm 1.55 \\
\hline & & Valor $\mathrm{p}$ & & \multicolumn{2}{|c|}{ NS } & \multicolumn{2}{|c|}{$<0.001$} & \multicolumn{2}{|c|}{ NS } \\
\hline & & $\mathrm{TE}$ & & \multicolumn{2}{|c|}{-0.07} & \multicolumn{2}{|c|}{-0.27} & \multicolumn{2}{|c|}{0.00} \\
\hline & Prácticas & \multirow[t]{2}{*}{ Grupos } & 1 & 1.65 & \pm 1.62 & 68.30 & \pm 12.83 & 0.70 & \pm 0.41 \\
\hline & & & 2 & 1.42 & \pm 1.66 & 74.43 & \pm 10.35 & 1.36 & \pm 2.13 \\
\hline & & \multicolumn{2}{|l|}{ Valor p } & \multicolumn{2}{|c|}{ NS } & \multicolumn{2}{|c|}{$<0.05$} & \multicolumn{2}{|c|}{ NS } \\
\hline & & \multicolumn{2}{|l|}{$\mathrm{TE}$} & \multicolumn{2}{|c|}{0.07} & & & & \\
\hline & Deporte & Grupos & 1 & 3.94 & \pm 3.91 & 106.03 & \pm 19.91 & 2.60 & \pm 3.39 \\
\hline & & & 2 & 1.52 & \pm 0.84 & 97.84 & \pm 11.83 & 1.36 & \pm 1.02 \\
\hline & & Valor p & & & & & & & $S$ \\
\hline & & TE & & & & & & & 24 \\
\hline & Salida 1 & Grupos & 1 & 1.37 & \pm 0.98 & 69.65 & \pm 8.35 & 0.26 & \pm 0.16 \\
\hline & & & 2 & 2.19 & \pm 1.84 & 78.50 & \pm 7.48 & 0.16 & \pm 0.25 \\
\hline & & Valor $\mathrm{p}$ & & & & & & & \\
\hline & & TE & & & & & & & 3 \\
\hline & Salida 2 & Grupos & 1 & 1.28 & \pm 1.04 & 65.99 & \pm 9.23 & 0.68 & \pm 0.72 \\
\hline & & & 2 & 1.51 & \pm 1.41 & 77.17 & \pm 13.02 & 1.08 & \pm 1.22 \\
\hline & & Valor $\mathrm{p}$ & & & & & & & \\
\hline & & $\mathrm{TE}$ & & & & & & & \\
\hline
\end{tabular}

Nota. Grupos: (1) Experimentados, (2) Noveles; M: Media; DE: Desviación estándar; TE: Tamańo del efecto; NS: No Significativo (p>.05).

\section{Discusión}

Los resultados de este trabajo han presentado diferencias significativas en la variable AcelT en dos de las cinco activida- des analizadas (Reposo y Deporte), siendo estas las dos únicas actividades en las que los bomberos no realizan la misma actividad. En estas actividades cada bombero dispone de cierta libertad para realizar, o no, la actividad que él consi- 
dere oportuna. Esto se debe a que, en el parque de bomberos de Cartagena, como se menciona en otro estudio realizado por Reilly (2006), son los propios bomberos los que de forma autónoma y voluntaria se encargan de su entrenamiento por la no presencia de la figura de un entrenador personal que coordine, asesore y planifique el entrenamiento físico de la plantilla del parque como aconseja Prieto et al. (2010). El mayor AcelT registrado en la actividad Deporte en el grupo 1 (experimentados) es debido a que estos prefieren la realización de actividades aeróbicas o bicicleta estática, mientras que el grupo 2 se centra más en un trabajo de musculación que requiere menor impacto para su realización.

Por otra parte, los resultados muestran que la respuesta al estrés (R-S|Ps) durante la jornada laboral de los bomberos varía en función de la actividad que se desempeña (tabla 1). En el grupo 2 registra mayor estrés que el grupo 1 en Prácticas y Salida de Emergencia ( $\mathrm{p}<.10$ y $\mathrm{p}<.01$, respectivamente). Los datos obtenidos coinciden con estudios anteriores que indican que los bomberos sin experiencia presentan mayores índices de estrés, además de estar más predispuestos a sufrir el síndrome de burnout (Moreno et al., 2006; Peñacoba et al., 2000). Por otro lado, existen estudios que han puesto de manifiesto que ciertas características personales, como la experiencia, modulan los efectos de los parámetros fisiológicos del estrés en la salud del individuo. Concretamente, este factor ha sido conceptualizado como atenuador en la respuesta del estrés laboral puesto que influye tanto en la percepción de la situación estresante como en la elección de la estrategia de afrontamiento (Schuler, 1980). Vivenciar reiteradamente ciertas situaciones puede reducir la incertidumbre y, consecuentemente disminuir el estrés. Por tanto, los resultados de este estudio se encuentran en concordancia con estas indicaciones. Además de la experiencia, una investigación realizada por de Miguel Calvo, Schweiger Gallo, De las Mozas Majano, y Hernández López (2011) expone que los participantes en un programa específico de entrenamiento manifestaron mayores niveles de bienestar y satisfacción el trabajo, menor grado de estrés percibido y obtuvieron una valoración de su rendimiento laboral más elevada que los participantes en el grupo control. Por tanto, no solo la experiencia es importante para disminuir el estrés, sino también la realización de programas de entrenamiento específicos que simulen las situaciones de estrés para conseguir un mayor rendimiento a posteriori.

Finalmente, respecto a la variable frecuencia cardiaca, los bomberos del grupo 2 han presentado mayores valores que los del grupo 1 en cinco de las seis actividades analizadas (Dormir, Reposo, Prácticas, Salida sin emergencia y Salida con emergencia), con la excepción de la categoría Deporte, en la cual el grupo 1 ha registrado valores superiores. Este comportamiento es similar al obtenido por López et al. (2018) que encuentran una optimización de las exigencias físicas y fisiológicas en remeros experimentados respecto a remeros nova- tos. Además, estudios en la literatura han encontrado que los bomberos con menor experiencia presentan mayores índices de estrés (Moreno et al., 2006; Peñacoba et al., 2000) y este efecto produce como consecuencia una modificación en la respuesta fisiológica a través del aumento de la frecuencia cardiaca (Gómez-Alcaina et al., 2013; Moya-Albiol et al., 2005). Otros factores que justifican esta diferencia son la edad del sujeto (Tsuji et al., 1996) y su entrenamiento. Lara et al. (2013) encuentra que los bomberos jóvenes presentan mayor masa muscular y este hecho produce, entre otras adaptaciones, un aumento de la frecuencia cardiaca de reposo (López, López, y Díaz, 2015).

\section{Conclusiones}

A partir de los resultados obtenidos en esta investigación, se extraen dos conclusiones principales: (1) Los bomberos con menos experiencia sufren un mayor estrés y un aumento de la frecuencia cardíaca ante salidas de urgencia, como incendios o accidentes de tráfico, y (2) No existe un control por parte de personal cualificado para el análisis de las exigencias físicas y psicológicas de la actuación de los bomberos para una planificación del entrenamiento y estrategias de afrontamiento ante la resolución de conflictos adaptadas a la situación real. Por tanto, futuras estudios deberían seguir las líneas de investigación propuestas a continuación:

1. Aumento del tamaño muestral. Aceptando un riesgo alfa de 0.05 y un riesgo beta de 0.2 en un contraste bilateral, se precisan 44 sujetos en el primer grupo y 44 en el segundo para detectar una diferencia igual o superior a 0.6 unidades. Además, se debería realizar una mayor duración de registro para que los datos de los resultados fuesen más fiables.

2. Modificar la agrupación por años de experiencia. Comparar bomberos con menos años de experiencia para poder comparar sus niveles de estrés con el de los bomberos con mayor experiencia con el objetivo de definir el tiempo de trabajo que se requiere para que los bomberos no presenten distintos índices de estrés, aunque debido a los resultados, se piensa que este estudio se encuentra cerca de ese periodo de tiempo.

3. Relacionar el estrés fisiológico con el estrés psicológico subjetivo percibido. Medir el estrés percibido por los bomberos y que se comparase este con el estrés fisiológico que experimentan en su labor, pues a lo largo de este estudio, se ha podido formular la hipótesis de que su percepción del estrés es inferior a la registrada fisiológicamente.

4. Intervención a través de un programa de entrenamiento físico y psicológico adaptado a las exigencias de la profesión. Su realización permitiría determinar si someter a los bomberos, sobre todo a aquellos con menos años de 
experiencia, a situaciones estresantes tanto física como psicológicamente semejantes a la situación real permiti- ría durante la intervención reducir el estrés y por tanto mejorar el rendimiento laboral.

\section{Bibliografía}

1. Avellaneda Pinzón, S. E., y Urbina, A. (2015). Capacidad aeróbica de bomberos aeronáuticos. Salud, Revista de la Universidad Industrial de Santander, 47(1), 61-67.

2. Bastida-Castillo, A., Gómez-Carmona, C. D., De la Cruz Sánchez, E., \& Pino-Ortega, J. (2018a). Accuracy, intra-and inter-unit reliability, and comparison between GPS and UWB-based position-tracking systems used for time-motion analyses in soccer. European journal of sport science, 18(4), 450-457. doi: 10.1080/17461391.2018.1427796

3. Bastida-Castillo, A., Gómez-Carmona, C., Hernández-Belmonte, A., \& Pino-Ortega, J. (2018b). Validez y fiabilidad de un dispositivo inercial (WIMU $\mathrm{PRO}^{\mathrm{TM}}$ ) para el análisis del posicionamiento en balonmano. E-balonmano.com: Revista de Ciencias del Deporte, 14(1), 9-16.

4. Bouzas, J. C., Ottoline, N. M., y Delgado, M. (2010). Aplicaciones de la frecuencia cardiaca máxima en la evaluación y prescripción de ejercicio. Apunts. Medicina de l'Esport, 45(168), 251-258. doi: 10.1016/j. apunts.2010.04.003

5. Cayo, J. E., y Chamorro, S. E. (2015). Enfermedades crónicas, la hipertensión arterial influenciada por cambios en el estilo de vida, en los pacientes que acudieron al Hospital Provincial General de Latacunga en el periodo Enero-Diciembre 2014 (Trabajo de grado). Facultad de Ciencias Médicas. Universidad Central del Ecuador, Quito, Ecuador.

6. Cormack, S. J., Mooney, M. G., Morgan, W., y McGuigan, M. R. (2013). Influence of neuromuscular fatigue on accelerometer load in elite Australian football players. International journal of sports physiology and performance, 8(4), 373-378. doi: 10.1123/ijspp.8.4.373

7. Curilem, G. C., Almagià, F. A., Yuing, F. T., y Rodríguez, R. F. (2014). Evaluación del Estado Psicobiotipológico en Bomberos: Parámetros de Salud y Recursos Anti Estrés. International Journal of Morphology, 32(2), 709-714. doi: 10.4067/S0717-95022014000200054

8. de Miguel Calvo, J. M., Schweiger Gallo, I., De las Mozas Majano, O., y Hernández López, J. M. (2011). Efecto del ejercicio físico en la productividad laboral y el bienestar. Revista de Psicología del Deporte, 20(2), 589-604.

9. De Vicente, M.A. (2005). Análisis bibliográfico de la profesión de bombero. Madrid: Instituto Nacional de Seguridad en el Trabajo.

10. García, H. D., Fantin, M. B., y Pardo, A. Y. (2016). Estrategias de afrontamiento al estrés en aspirantes a Policía de la ciudad de San Luis. Revista de Psicología Universidad de Antioquia, 8(1), 23-34.

11. Gómez-Alcaina, B., Montero-Marín, J., Demarzo, M. M., Pereira, J. P., y García-Campayo, J. (2013). Utilidad de los marcadores biológicos en la detección precoz y prevención del síndrome de burnout. Revista de Psicopatología y Psicología Clínica, 18(3), 245-253. doi: 10.5944/rppc. vol.18.num.3.2013.12924

12. Gómez-Carmona, C. D., Bastida-Castillo, A., García-Rubio, J., Ibáñez, S. J., y Pino-Ortega, J. (2018). Static and dynamic reliability of WIMU PRO ${ }^{\mathrm{TM}}$ accelerometers according to anatomical placement. Proceedings of the Institution of Mechanical Engineers, Part P: Journal of Sports Engineering and Technology, Epub: Ahead of print. doi: $10.1177 / 1754337118816922$

13. Guidotti, T. (2001). Capítulo 95. Servicios de seguridad y emergencia. En Organización Internacional del Trabajo (Ed.), Enciclopedia de salud y seguridad en el trabajo (pp. 95.2 - 95.22). Madrid: Organización Internacional del Trabajo.

14. Kunze, K., Bahle, G., Lukowicz, P., y Partridge, K. (2010). Can magnetic field sensors replace gyroscopes in wearable sensing applications? En 2010 International Symposium on Wearable Computers (ISWC), (pp.
1-4). IEEE. Recuperado a partir de http://ieeexplore.ieee.org/abstract/ document/5665859/

15. Laca, F. A., Mejía, J. C., y Condra, J. M. (2014). Propuesta de un modelo para evaluar el bienestar laboral como componente de la salud mental. Psicología y salud, 16(1), 87-92.

16. Lara, A. J., García, J. M., Torres-Luque, G., y Zagalaz, M. L. (2013). Análisis de la condición física en bomberos en función de la edad. Apunts. Medicina de l'Esport, 48(177), 11-16. doi: 10.1016/j. apunts.2011.11.006

17. López, G. F., López, L., y Díaz, A. (2015). Composición corporal y variabilidad de la frecuencia cardiaca: relaciones con edad, sexo, obesidad y actividad física. Sportk: revista euroamericana de ciencias del deporte, 4(2), 33-40. doi: 10.6018/242921

18. López Sánchez, G. F., Smith, L., Díaz Suárez, A., Towner, A., \& Gordon, D. (2018). Do novice and experienced rowers adopt different pacing strategies and do their physiological and metabolic responses show optimisation? Sportk: revista euroamericana de ciencias del deporte, 7(1), 165-174. doi: 10.6018/322031

19. López, J., Villa, J. G., Rodríguez, J. A., García, J., Moreno, S., Ávila, C., y Pernía, R. (2006). Estudio de los factores condicionantes del rendimiento físico del personal especialista en la extinción de incendios forestales: pruebas de aptitud física de selección de personal. En Actas de 4 a Conferencia Internacional sobre Incendios Forestales (pp. 157-165). Recuperado a partir de http://www.fire.uni-freiburg.de/sevilla-2007/ contributions/doc/cd/SESIONES_TEMATICAS/ST6/Lopez-Satue_ et_al_TRAGSA_CLM2.pdf

20. Mateo-March, M., Rodríguez-Pérez, M. A., Costa, R., Sánchez-Muñoz, C., Casimiro-Andújar, A. J., y Zabala, M. (2013). Efecto de un programa de intervención sobre el estrés percibido, autoestima y rendimiento en jóvenes pilotos de motociclismo de elite. Revista de Psicología del Deporte, 22(1), 125-133.

21. Molina-Carmona, I., Gomez-Carmona, C. D., Bastida Castillo, A. y Pino-Ortega, J. (2018). Validez del dispositivo inercial WIMU PROTM para el registro de la frecuencia cardíaca en un test de campo. Sport-TK: Revista Euroamericana de Ciencias del Deporte, 7, 81-86. doi: $10.6018 / 321921$

22. Molinero, O., Salguero, A., y Márquez, S. (2012). Estrés-recuperación en deportistas y su relación con los estados de ánimo y las estrategias de afrontamiento. Revista de Psicología del Deporte, 21(1), 163-170.

23. Moreno, B., Morett, N. I., Rodríguez, A., y Morante, M. E. (2006) La personalidad resistente como variable moduladora del síndrome de burnout en una muestra de bomberos. Psicothema, 18(3), 413-418.

24. Moya-Albiol, L., Serrano, M. ángel, González-Bono, E., RodríguezAlarcón, G., y Salvador, A. (2005). Respuesta psicofisiológica de estrés en una jornada laboral. Psicothema, 17(2), 205-211.

25. Naranjo, J., y De La Cruz, B. (2010). La entropía y la irreversibilidad temporal multiescala en el análisis de sistemas complejos en fisiología humana. Revista Andaluza de Medicina del Deporte, 3(1), 29-32.

26. O’Donovan, K. J., Kamnik, R., O’Keeffe, D. T., y Lyons, G. M. (2007). An inertial and magnetic sensor-based technique for joint angle measurement. Journal of Biomechanics, 40(12), 2604-2611. doi: 10.1016/j. jbiomech.2006.12.010

27. Paris, L. (2016). Estrategias de afrontamiento del estrés asistencial y satisfacción laboral-personal en médicos y enfermeros. Revista Interamericana de Psicología Ocupacional, 26(2), 7-21.

28. Peńacoba, C., Díaz, L., Goiri, E., y Vega, R. (2000). Estrategias de afrontamiento ante situaciones de estrés: Un análisis comparativo entre 
bomberos con y sin experiencia. Revista de Psicología del Trabajo y de las Organizaciones, 16(3), 341-356.

29. Prieto, J. A., Del Valle, M., Montoliú, M. Á., Martínez, P. C., Nistal, P., y González, V. (2010). Relación entre la percepción de la capacidad aeróbica y el VO2máx en bomberos. Psicothema, 22(1), 131-136.

30. Real Academia Espańola. (2014). Diccionario de la lengua española (23. ${ }^{a}$ ed.). Consultado en http://www.rae.es/rae.html

31. Reilly, T. (2006). Occupational fitness standards for beach lifeguards. Phase 2: the development of an easily administered fitness test. Оссираtional Medicine, 56(1), 12-17. doi: 10.1093/occmed/kqi168

32. Rosales-Soto, G., Corsini-Pino, R., Monsálves-Álvarez, M., y YáńezSepúlveda, R. (2016). Respuesta del balance simpático-parasimpático de la variabilidad de la frecuencia cardíaca durante una semana de entrenamiento aeróbico en ciclistas de ruta. Revista Andaluza de Medicina del Deporte, 9(4), 143-147. doi: 10.1016/j.ramd.2015.07.002
33. Rotger, D. (1994). El estrés de los bomberos, Mapfre Seguridad, 35, 11-21. 34. Rotger, D. (1997). Mejorando la toma de decisiones en situaciones de emergencia. El entrenamiento de los bomberos. Mapfre Seguridad, 67, 25-31.

35. Sabido, R., Gómez, J., Barbado, D., y Gómez-Valadés, J. M. (2013). Rendimiento en una prueba específica de bomberos y su relación con tests físicos. European Journal of Human Movement, 30, 23-35.

36. Salvador, A., y González-Bono, E. (1995). Trastornos psicológicos asociados al estrés laboral. Ansiedad y estrés, 1(2), 157-171.

37. Topa, G., Fernández, I., y Lisbona, A. (2005). Ruptura de contrato psicológico y burnout en equipos de intervención y catástrofes. Ansiedad y estrés, 11, 2-3.

38. Tsuji, H., Venditti, F. J., Manders, E. S., Evans, J. C., Larson, M. G., Feldman, C. L., y Levy, D. (1996). Determinants of heart rate variability. Journal of the American College of Cardiology, 28(6), 1539-1546. 